\title{
Adsorption Behavior of Avidin and Streptavidin on the Gold Electrodes Modified with Thiol Monolayers with Different Hydrophobicity and Polarity
}

\author{
Qiang CHEN, Jun-ichi ANZAI* and Tetsuo OSA
}

Received June 1, 1996 ; Accepted August 19, 1996

\section{INTRODUCTION}

Avidin and streptavidin are known to bind biotin very strongly and selectively (binding constant:1015 $\left.\left.\mathrm{M}^{-1}\left(\mathrm{M}=\mathrm{mol} \mathrm{dm}^{-3}\right)\right)^{1}\right)$. For this reason, an avidin-biotin system has been used to immobilize biotin-labeled enzymes on the surface of metal and carbon electrodes for the preparation of electrochemical enzyme sensors 2,3 ). It has been reported that avidin and streptavidin can be adsorbed irreversibly on the solid surfaces4). However, the effects of surface properties on the adsorption behavior of avidin and streptavidin is still equivocal. 3,4) The present paper reports the adsorption behavior of avidin and streptavidin on the gold electrodes modified with self-assembled thiol monolayers with different hydrophobicity and polarity. The surface properties of thiol-modified electrodes often affect the biological activity of immobilized proteins 5 ).

\section{EXPERIMENTAL}

Avidin and streptavidin were purchased from Calzyme Lab. Inc. (USA) and Southern Biotech. Assoc. Inc. (USA), respectively. Biotin-labeled glucose oxidase (GOx) was obtained from Sigma Co. (USA). Thiol compounds used are propanethiol (PT), 2-hydroxyethanethiol (2-HET), 2-aminoethanethiol (2-AET), thioglycolic acid (TGA) and sodium 2mercaptoethanesulfonate (2-MES).

The modification of gold electrode with thiol monolayers was carried out as in a usual manner6); a gold-sputtered quartz resonator (area of gold disk; 4 $\mathrm{mm}$ diameter) or a gold disk electrode $(1.6 \mathrm{~mm}$ diameter) was immersed in one of the thiol solutions in ethanol $(10 \mathrm{mM})$ for $4 \mathrm{~h}$. The thiol monolayermodified $\mathrm{Au}$ electrodes were rinsed thoroughly with ethanol and working buffer. The surface of PT- and

\footnotetext{
Pharmaceutical Institute, Tohoku University (Aobayama, Sendai 980-77, Japan)

Key Words: Quartz-crystal microbalance, Avidin, Streptavidin, Enzyme sensor
}

2-HT-modified electrodes should be hydrophobic and hydrophilic, respectively. It is reasonable to assume that, in neutral pH, the surfaces of the TGA- and 2MES-modified electrodes are negatively charged, while the 2-AET-modified electrode is positively charged 7,8 ).

A quartz-crystal microbalance (QCM) with a 9 MHz AT-cut quartz resonator (QCA-912, Seiko EG \& $G$ Co.) was used to monitor the adsorption of avidin and stereptavidin. In the $9 \mathrm{MHz}$ AT-cut device, one nanogram of mass loading induces a frequency changes of $-0.91 \mathrm{~Hz}^{9}$ ). An amperometric response of GOX-modified electrodes was measured at $0.6 \mathrm{Vvs}$. $\mathrm{Ag} / \mathrm{AgCl}$ in the presence of glucose. The GOx-modified electrodes were prepared as reportedly3). All measurements were performed at room temperature $\left(18-20^{\circ} \mathrm{C}\right)$.

\section{RESULTS AND DISCUSSION}

Figure 1 shows the frequency changes $(\Delta F)$ caused by the adsorption of avidin onto the thiol monolayer-modified and unmodified Au electrodes in a phosphate buffered saline (PBS). In all cases, the resonant frequency (F) decreases on addition of avidin in the buffer solution (final concentration of avidin; $10 \mu \mathrm{g} / \mathrm{ml}$ ). This indicates that avidin molecules are adsorbed onto the surface of the $\mathrm{Au}$ electrodes to increase the mass loading $(\Delta M)$ on the quartz crystal. It should be noted here that the $\Delta F$ values depend on the type of thiol monolayers. Avidin adsorbs more efficiently to the negatively charged surfaces of 2-MES- and TGA-modified electrodes than to the other positively charged and electrically neutral surfaces (2-AET, PT, and 2-HET monolayers). This is probably due to the electrostatic force of attraction between positively charged avidin molecules (pI of avidin; 9-101)) and the negatively charged surface of 2-MES and TGA monolayers. This view is supported by the fact that, in a phosphate buffer of higher ionic strength which contains $1 \mathrm{M}$ $\mathrm{NaCl}$, the adsorption of avidin to the 2-MES- 


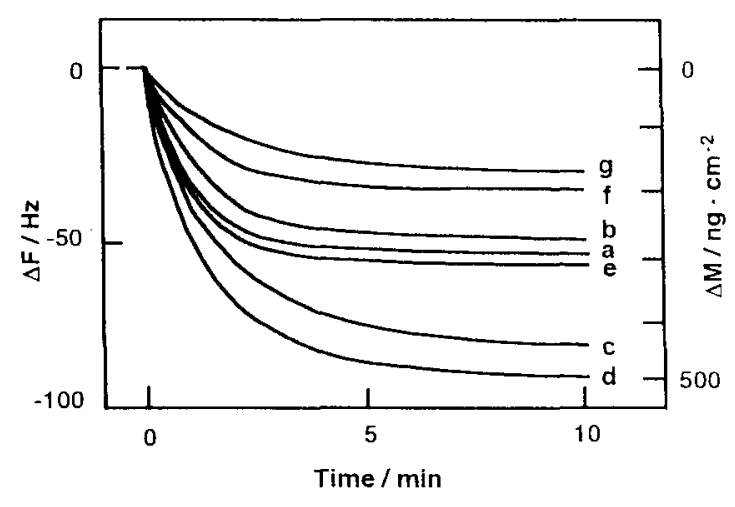

Fig. 1 Frequency changes of thiol-modified QCM on adsorption of avidin in PBS. Thiol monolayer: none (a), 2-AET (b), TGA (c), 2-MES (d, g), PT (e), and 2-HET (f). A phosphate buffer of higher ionic strength was used in $(\mathrm{g})$.

modified electrode is reduced to ca. $180 \mathrm{ng} / \mathrm{cm}^{2}$ as compared with ca. $500 \mathrm{ng} / \mathrm{cm}^{2}$ in PBS containing ca. $0.14 \mathrm{M} \mathrm{NaCl}$. Another feature is that the adsorption of avidin is less significant to the hydrophilic 2-HET surface than to the hydrophobic PT monolayer, suggesting the partial participation of hydrophobic interaction in the adsorption of avidin. If the electrode surface is covered with a monomolecular layer of avidin, the loading of avidin should be 340 $510 \mathrm{ng} / \mathrm{cm}^{2}$ depending on the orientation of avidin molecules on the surface, calculating from the molecular dimensions of $4.0 \mathrm{~nm} \times 5.5 \mathrm{~nm} \times 6.0 \mathrm{~nm}$ and molecular mass of ca. 68,0001). According to the QCM data (Fig. 1), avidin seems to form monomolecular layer on the surface of 2-MES- and TGA-modified electrodes, though the orientation of avidin molecules on the surface is ambiguous.

The adsorption behavior of streptavidin onto the thiol-modified electrodes has been also studied using QCM and found that the adsorption is far less significant than avidin irrespective of the type of thiol monolayers. The loading of streptavidin is $40 \mathrm{ng} / \mathrm{cm}^{2}$ or less for all monolayers tested. This difference in the adsorption behavior is in accord with the general trends that non-specific adsorption of streptavidin is lower than that of avidin, probably due to the nonglycosylated nature of streptavidin 1 ).

From the viewpoint of biosensor application of the avidin-modified Au electrodes, it is required that the avidin molecules retain their binding ability to biotin at the electrode surface. To check the binding ability of avidin, the avidin-modified electrodes were treated with biotin-labeled glucose oxidase (GOx) and their enzymatic activity was measured amperometrically (Fig. 2). The GOx-modified electrodes show amperometric response to glucose, confirming that

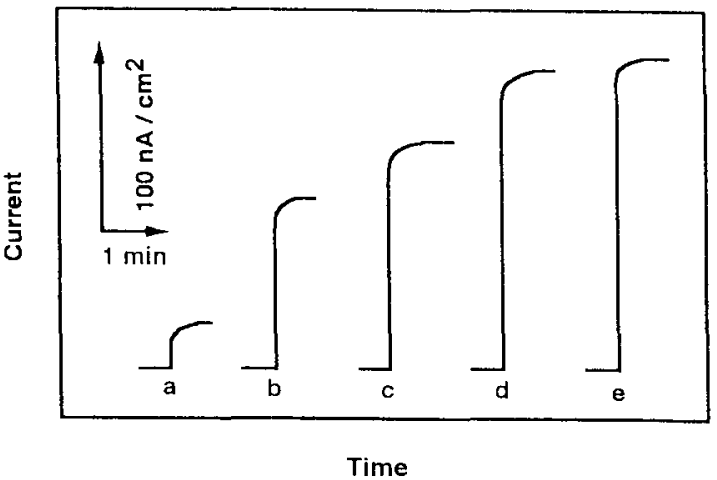

Fig. 2 Response of GOx-modified Au electrodes to 6 $\mathrm{mM}$ glucose in PBS. Electrode potential: $0.6 \mathrm{~V} v s$. $\mathrm{Ag} / \mathrm{AgCl}$. Thiol monolayer: 2-HET (a), 2-AET (b), PT (c), TGA (d), and 2-MES (e).

the amperometric response to glucose, confirming thatthe biotin-labeled GOx is immobilized on the electrodesurfaces. In other words, avidin molecules are still active to bind biotin residue at the surface of the thiol monolayers. The output current depends significantly on the type of thiol monolayers of the electrodes. The output current is in the order of 2 MES- > TGA- > PT- > 2-AET- > 2-HET-modified electrode, which is in accord with the order of the loading of avidin as measured with QCM (Fig. 1).

Thus, is is concluded that 1)avidin is adsorbed more efficiently on the surface of negatively charged thiol monolayers without loss of the binding ability to biotin, while the adsorption of streptavidin onto the thiol monolayers is rather suppressed.

The present work was supported in part by Grant-in-Aid (No.08458282) from the Ministry of Education, Science, Sports and Culture of Japan.

\section{REFERENCES}

1) M.Wilchek and E.A.Bayer, Anal.Biochem., 171, 1(1988).

2) P.Pantano and W.G.Kuhr, Anal.Chem., 65, 623(1993).

3) T.Hoshi, J.Anzai and T.Osa, Anal.Chem., 67, 770(1995).

4) R.C.Ebersole, J.A.Miller, J.R.Moran and M.D. Ward, J.Am.Chem.Soc., 112, 3239(1990).

5) J.Anzai, B.Guo and T.Osa, Bioelectrochem. Bioenerg., 40, 35(1996).

6) M.D.Porter, T.B.Bright, D.L.Allara and C.E.D. Chidsey, J.Am.Chem.Soc., 109,3559(1987).

7) M.Cefola, A.S.Tompa, A.V.Celiano and P.S.Gentile, Inorg.Chem., 1, 290(1962).

8) Y.Tsuchitani, T.Ando and K.Ueno, Bull.Chem.Soc.Jpn., 36, 1534(1963).

9) G.Sauerbrey, Z.Phys., 155, 206(1959). 